\title{
Minimal time for the bilinear control of Schrödinger equations
}

\author{
Karine Beauchard, , Jean-Michel Coron ${ }^{\dagger}$ Holger Teismann ${ }^{\ddagger}$
}

\begin{abstract}
We consider a quantum particle in a potential $V(x)\left(x \in \mathbb{R}^{N}\right)$ subject to a (spatially homogeneous) time-dependent electric field $E(t)$, which plays the role of the control. Under generic assumptions on $V$, this system is approximately controllable on the $L^{2}\left(\mathbb{R}^{N}, \mathbb{C}\right)$-sphere, in sufficiently large times $T$, as proved by Boscain, Caponigro, Chambrion and Sigalotti 7 . In the present article, we show that this approximate controllability result is false in small time. As a consequence, the result by Boscain et al. is, in some sense, optimal with respect to the control time $T$.
\end{abstract}

\section{Introduction}

\subsection{Main result}

In this article, we consider quantum systems whose dynamics can be described by a linear Schrödinger equation of the form

$$
\begin{cases}i \partial_{t} \psi(t, x)=\left(-\frac{1}{2} \Delta+V(x)-\langle E(t), x\rangle\right) \psi(t, x), & (t, x) \in(0, T) \times \mathbb{R}^{N}, \\ \psi(0, x)=\psi_{0}(x), & x \in \mathbb{R}^{N} .\end{cases}
$$

Here, $N \in \mathbb{N}^{*}$ is the space dimension, $\langle.,$.$\rangle is the usual scalar product on \mathbb{R}^{N}$, $V: x \in \mathbb{R}^{N} \rightarrow \mathbb{R}, E: t \in(0, T) \rightarrow \mathbb{R}^{N}$ and $\psi:(t, x) \in(0, T) \times \mathbb{R}^{N} \rightarrow \mathbb{C}$ are a static potential, a time-dependent electric field, and the wave function, respectively. This equation represents a quantum particle in the potential $V$ subject to the electric field $E(t)$. Planck's constant and the particle mass have been set to one.

System (1) is a control system in which the state is the wave function $\psi$, that belongs to the unitary $L^{2}\left(\mathbb{R}^{N}, \mathbb{C}\right)$-sphere, denoted by $\mathcal{S}$; and the control is the electric field $E$. Such systems have applications in modern technologies such as Nuclear Magnetic Resonance, quantum chemistry and quantum information science. The expression 'bilinear control' refers to the 'bilinear' nature w.r.t. $(E, \psi)$ of the term $\langle E(t), x\rangle \psi$.

\footnotetext{
*CMLS, Ecole Polytechnique, 91128 Palaiseau Cedex, France, email: Karine.Beauchard@math.polytechnique.fr (corresponding author)

†Sorbonne Universités, UPMC Univ Paris 06, UMR 7598, Laboratoire Jacques-Louis Lions, F-75005, Paris, France email: coron@ann.jussieu.fr

${ }^{\ddagger}$ Department of Mathematics and Statistics, Acadia University, Wolfville, NS, Canada email: hteisman@acadiau.ca
} 
We are interested in the minimal time required to achieve approximate controllability of system (1). Since in (1) decoherence is neglected, in realistic scenarios the model may only be applicable for small times $t$ (typically on the order of several periods of the ground state). Thus, to be practically relevant, controllability results need to be valid for time intervals for which equation (1) remains a reasonable model. Therefore quantification of the minimal control time is an important issue.

First, we recall a classical well-posedness result [16], which we quote from [10. We consider potentials $V$ that are smooth and subquadratic, i.e.

$$
V \in C^{\infty}\left(\mathbb{R}^{N}\right) \text { and, } \forall \alpha \in \mathbb{N}^{N} \text { such that }|\alpha| \geqslant 2, \partial_{x}^{\alpha} V \in L^{\infty}\left(\mathbb{R}^{N}\right) .
$$

Proposition 1. Consider $V$ satisfying assumption (2) and $E \in L_{l o c}^{\infty}\left(\mathbb{R}, \mathbb{R}^{N}\right)$. There exists a strongly continuous map $(t, s) \in \mathbb{R}^{2} \mapsto U(t, s)$, with values in the set of unitary operators on $L^{2}\left(\mathbb{R}^{N}, \mathbb{C}\right)$, such that

$$
U(t, t)=I d, \quad U(t, \tau) U(\tau, s)=U(t, s), \quad U(t, s)^{*}=U(s, t)^{-1}, \quad \forall t, \tau, s \in \mathbb{R}
$$

and for every $t, s \in \mathbb{R}, \varphi \in L^{2}\left(\mathbb{R}^{N}, \mathbb{C}\right)$, the function $\psi(t, x):=U(t, s) \varphi(x)$ solves the first equation of (11) with initial condition $\psi(s, x)=\varphi(x)$.

For $V$ satisfying (2), we introduce the operator

$$
D\left(A_{V}\right):=\left\{\varphi \in L^{2}\left(\mathbb{R}^{N}\right) ;-\Delta \varphi+V(x) \varphi \in L^{2}\left(\mathbb{R}^{N}\right)\right\}, A_{V} \varphi:=-\frac{1}{2} \Delta \varphi+V(x) \varphi
$$

For appropriate potentials $V$, approximate controllability of (1) in $\mathcal{S}$ (possibly in large time) is a corollary of a general result by Boscain, Caponigro, Chambrion, Mason and Sigalotti (the original proof of [11] is generalized in [7]; inequality (4) below is proved in [11, Proposition 4.6]; see also [8] for a survey of results in this area).

Theorem 1. We assume that

- there exists a Hilbert basis $\left(\phi_{k}\right)_{k \in \mathbb{N}}$ of $L^{2}\left(\mathbb{R}^{N}, \mathbb{C}\right)$ made of eigenvectors of $A_{V}: A_{V} \phi_{k}=\lambda_{k} \phi_{k}$ and $x \phi_{k} \in L^{2}\left(\mathbb{R}^{N}\right), \forall k \in \mathbb{N}$,

- $\int_{\mathbb{R}^{N}} x \phi_{j}(x) \phi_{k}(x) d x=0$ for every $j, k \in \mathbb{N}$ such that $\lambda_{j}=\lambda_{k}$ and $j \neq k$,

- for every $j, k \in \mathbb{N}$, there exists a finite number of integers $p_{1}, \ldots, p_{r} \in \mathbb{N}$ such that

$$
p_{1}=j, \quad p_{r}=k, \quad \int_{\mathbb{R}^{N}} x \phi_{p_{l}}(x) \phi_{p_{l+1}}(x) d x \neq 0, \forall l=1, \ldots, r-1,
$$

$\left|\lambda_{L}-\lambda_{M}\right| \neq\left|\lambda_{p_{l}}-\lambda_{p_{l+1}}\right|, \forall 1 \leqslant l \leqslant r-1, L, M \in \mathbb{N}$ with $\{L, M\} \neq\left\{p_{l}, p_{l+1}\right\}$.

Then, for every $\epsilon>0$ and $\psi_{0}, \psi_{f} \in \mathcal{S}$, there exist a time $T>0$ and $a$ piecewise constant function $u:[0, T] \rightarrow \mathbb{R}$ such that the solution of (1) satisfies

$$
\left\|\psi(T)-\psi_{f}\right\|_{L^{2}\left(\mathbb{R}^{N}\right)}<\epsilon .
$$

Moreover, for every $\delta>0$, the existence of a piecewise constant function $u$ : $[0, T] \rightarrow(-\delta, \delta)$ such that the solution of (1) satisfies (3) implies that

$$
T \geqslant \frac{1}{\delta} \sup _{k \in \mathbb{N}} \frac{||\left\langle\phi_{k}, \psi_{0}\right\rangle|-|\left\langle\phi_{k}, \psi_{f}\right\rangle||-\epsilon}{\left\|B \phi_{k}\right\|} .
$$


To prove this statement, the authors use finite dimensional techniques applied to the Galerkin approximations of equation (11). They also prove an estimate on the $L^{1}$-norm of the control [7, Proposition 2.8] and approximate controllability in the sense of density matrices [7, Theorem 2.11].

In Theorem 1, the time $T$ is not known a priori and may be large. Note that the lower bound on the control time in (4) goes to zero when $\delta \rightarrow+\infty$. Thus, approximate controllability in arbitrarily small time (allowing potentially large controls) is an open problem. The goal of this article is to prove that, for potentials $V$ satisfying (2), approximate controllability does not hold in arbitrarily small time, even with large controls, as stated in the following theorem.

Theorem 2. Consider $V$ satisfying assumption (2). Let $b>0, x_{0}, \dot{x}_{0} \in \mathbb{R}^{N}$ and $\psi_{0} \in \mathcal{S}$ be defined by

$$
\psi_{0}(x):=\frac{b^{N / 4}}{C_{N}} e^{-\frac{b}{2}\left\|x-x_{0}\right\|^{2}+i\left\langle\dot{x}_{0}, x-x_{0}\right\rangle}
$$

where

$$
C_{N}:=\left(\int_{\mathbb{R}^{N}} e^{-\|y\|^{2}} d y\right)^{1 / 2} .
$$

Let $\psi_{f} \in \mathcal{S}$ a state that does not have a Gaussian profile in the sense that

$\left|\psi_{f}().\right| \neq \frac{\operatorname{det}(S)^{1 / 4}}{C_{N}} e^{-\frac{1}{2}\|\sqrt{S}(.-\gamma)\|^{2}}, \quad \forall \gamma \in \mathbb{R}^{N}, S \in \mathcal{M}_{N}(\mathbb{R})$ symmetric positive.

Then there exist $T^{* *}=T^{* *}\left(\left\|V^{\prime \prime}\right\|_{\infty},\left\|V^{(3)}\right\|_{\infty}, b, \psi_{f}\right)>0$ and $\delta=\delta\left(\left\|V^{\prime \prime}\right\|_{\infty}, b, \psi_{f}\right)>$ 0 such that, for every $E \in C_{p w}^{0}\left([0, T], \mathbb{R}^{N}\right)$ (piecewise continuous functions $\left.[0, T] \rightarrow \mathbb{R}^{N}\right)$, the solution $\psi$ of (11) satisfies

$$
\left\|\psi(t)-\psi_{f}\right\|_{L^{2}\left(\mathbb{R}^{N}\right)}>\delta, \quad \forall t \in\left[0, T^{* *}\right] .
$$

In particular, if $V$ satisfies (2) and the assumptions of Theorem11(which hold generically, this fact may be proved as in [18), then system (1) is approximately controllable in $\mathcal{S}$ in large time but not in small time $T<T^{* *}$. In this sense, Theorem 1 is optimal with respect to the time of control. A characterization of the minimal time required for $\epsilon$-approximate controllability is an open problem.

\subsection{Bibliographical comments}

\subsubsection{Small-time control and minimal time for ODEs and PDEs}

In 15, D'Alessandro considers (generalizations of) Schrödinger ODEs and the controllability of their resolvent. He characterizes the set of states reachable in arbitrary time from the identity of the group. In particular, this set may not be the whole compact matrix Lie group even if the system is controllable. Then, in [1], Agrachev and Chambrion prove an estimation of the minimal time for the global approximate controllability. Such an estimate for PDE (1) is a widely open problem.

In [9], Boussaïd, Caponigro and Chambrion present an example of bilinear conservative system in infinite dimension for which approximate controllability holds in arbitrary small times. This situation is in contrast with the finite dimensional case discussed above and with Theorem 2 


\subsubsection{Minimal time for local exact controllability with small controls}

A different notion of 'minimal time' is investigated in 4. This article focuses on exact controllability and small controls to realize small motions whereas the present article investigates approximate controllability, large controls $E$ and large motions. Generalizing [14, the authors of [4] describe a general scenario for local exact controllability (with small controls) to hold in large time, but not in small time. This positive minimal time is related to the loss of directions of the linearized system and the behaviour of the second order term in the power series expansion of the solution.

\subsection{Notations}

Denote by $\mathcal{M}_{N}(\mathbb{K})$ the set of $N \times N$ matrices with coefficients in $\mathbb{K}=\mathbb{R}$ or $\mathbb{C}$ and $I_{N}$ its identity element; $\operatorname{Tr}(M)$ the trace of a matrix $M \in \mathcal{M}_{N}(\mathbb{C})$; $\mathcal{S}_{N}(\mathbb{R})$ (resp. $\mathcal{S}_{N}^{+}(\mathbb{R})$ ) the set of symmetric matrices (resp. positive symmetric matrices) in $\mathcal{M}_{N}(\mathbb{R}) ; A \leqslant B$ when $A, B \in \mathcal{S}_{N}(\mathbb{R})$ and $B-A \in \mathcal{S}_{N}^{+}(\mathbb{R}) ;\|$.$\| the$ Euclidean norm on $\mathbb{R}^{N}$ and the associated operator norm on $\mathcal{M}_{N}(\mathbb{R}) ; \dot{x}(t):=$ $\frac{d x}{d t}(t), \ddot{x}(t):=\frac{d^{2} x}{d t^{2}}(t)$, for a function $x$ of the variable $t ; C_{p w}^{0}\left([0, T], \mathbb{R}^{N}\right)$ the piecewise continuous functions $[0, T] \rightarrow \mathbb{R}^{N} ;\langle x, y\rangle:=\sum_{i=1}^{N} x_{i} y_{i}$, for every $x=$ $\left(x_{1}, x_{2}, \ldots, x_{n}\right) \in \mathbb{C}^{N}, y=\left(y_{1}, y_{2}, \ldots, y_{n}\right) \in \mathbb{C}^{N} ;$ and $\mathcal{S}$ the unit sphere in $L^{2}\left(\mathbb{R}^{N}, \mathbb{C}\right)$.

\section{Proof of Theorem 2}

Let $V$ satisfying (2), $b>0, x_{0}, \dot{x}_{0} \in \mathbb{R}^{N}$ and $\psi_{0}$ defined by (5).

Our strategy to prove Theorem 2, outlined in [21, is semi-classical: it relies on Gaussian approximate solutions that are localized around classical trajectories. They are called 'trajectory-coherent states' (TCS) and were originally introduced by Bagrov at al. [2, 5, 6, 3, (for recent and comprehensive mathematical treatments, see [20, 13]). They generalize the well-known explicit solutions for the harmonic oscillator potential $V(x)=x^{2}$ (see e.g. [17, 12, 19]) and may also be viewed as generalized WKB states.

The approximate solutions $\widetilde{\psi}=\widetilde{\psi}(t, x)$ (defined in eq. (13) below) depend on functions $x_{c}: \mathbb{R} \rightarrow \mathbb{R}^{N}$ and $Q: \mathbb{R} \rightarrow \mathcal{M}_{N}(\mathbb{C})$, which satisfy the ODEs (6) below. The vector function $x_{c}(t)$ is the classical (controlled) trajectory satisfying Newton's equation of motion (6), which includes the control field $E(t)$.

The remainder of this section is organized as follows. In Section 2.1, we prove a preliminary result for the solutions $Q(t)$ of (6) . In Section 2.2, we introduce the explicit approximate solution $\widetilde{\psi}$ and prove that the error $\|\psi-\widetilde{\psi}\|_{L^{\infty}\left((0, T), L^{2}\left(\mathbb{R}^{N}\right)\right)}$ can be bounded uniformly with respect to $E \in C_{p w}^{0}\left(\mathbb{R}, \mathbb{R}^{N}\right)$. Finally, Section 2.3 contains the proof of Theorem 2 


\subsection{The ODE for $Q(t)$}

For $E \in C_{p w}^{0}\left(\mathbb{R}, \mathbb{R}^{N}\right)$, we introduce the maximal solutions $x_{c} \in C^{1} \cap C_{p w}^{2}\left(\mathbb{R}, \mathbb{R}^{N}\right)$ and $Q \in C^{1}\left(\left(T^{-}, T^{+}\right), \mathcal{M}_{N}(\mathbb{C})\right)$ of

$$
\left\{\begin{array} { l } 
{ \frac { d ^ { 2 } x _ { c } } { d t ^ { 2 } } ( t ) + \nabla V [ x _ { c } ( t ) ] = E ( t ) , } \\
{ x _ { c } ( 0 ) = x _ { 0 } , } \\
{ \frac { d x _ { c } } { d t } ( 0 ) = \dot { x } _ { 0 } }
\end{array} \quad \left\{\begin{array}{l}
\frac{d Q}{d t}(t)+Q(t)^{2}+V^{\prime \prime}\left[x_{c}(t)\right]=0 \\
Q(0)=i b I_{N}
\end{array}\right.\right.
$$

where $\nabla V$ and $V^{\prime \prime}$ denote the gradient and Hessian matrix of $V$, respectively. Note that $x_{c}$ is defined for every $t \in \mathbb{R}$ because $\nabla V$ is globally Lipschitz by assumption (2); and the complex coefficient matrix $Q(t)$ is symmetric for every $t \in\left(T^{-}, T^{+}\right)$, so $Q_{2}(t):=\Im[Q(t)]$ is symmetric as well. A priori, the maximal interval $\left(T^{-}, T^{+}\right)$may depend on $E$.

Proposition 2. There exists $T^{*}=T^{*}\left(b,\left\|V^{\prime \prime}\right\|_{\infty}\right)>0$ such that, for every $E \in C_{p w}^{0}\left(\mathbb{R}, \mathbb{R}^{N}\right), Q(t)$ is defined for every $t \in\left[0, T^{*}\right]$ (i.e. $T^{+}>T^{*}$ ) and

$$
\frac{b}{2} I_{N} \leqslant Q_{2}(t) \leqslant \frac{3 b}{2} I_{N}, \text { for every } t \in\left[0, T^{*}\right]
$$

Proof of Proposition 2, Let $T^{*}=T^{*}\left(b,\left\|V^{\prime \prime}\right\|_{\infty}\right)>0$ be such that

$$
t\left[1+b e^{4 t}+\left\|V^{\prime \prime}\right\|_{\infty}\right]<1 \quad \text { and } \quad 2 t e^{2 t} \leqslant \frac{1}{2}, \quad \forall t \in\left[0, T^{*}\right] .
$$

Step 1: Equations satisfied by $Q_{1}(t):=\Re[Q(t)]$ and $Q_{2}(t):=\Im[Q(t)]$. Since $V^{\prime \prime}$ is real, (6) implies, on $\left(T^{-}, T^{+}\right)$

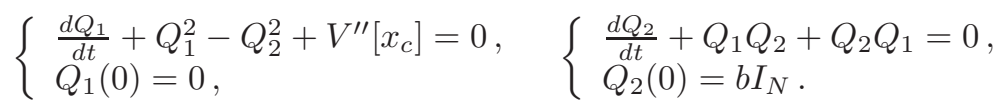

By Gronwall lemma and (9),

$$
\left\|Q_{2}(t)\right\| \leqslant b e^{2 \int_{0}^{t}\left\|Q_{1}(s)\right\| d s}, \quad \forall t \in\left[0, T^{+}\right) .
$$

Step 2: We prove that $T^{+}>T^{*}$ for every $E \in C_{p w}^{0}\left(\mathbb{R}, \mathbb{R}^{N}\right)$. Working by contradiction, we assume the existence of $E \in C_{p w}^{0}\left(\mathbb{R}, \mathbb{R}^{N}\right)$ such that $T^{+}<T^{*}$. In particular $T^{+}$is finite, thus $Q(t)$ explodes as $t \rightarrow T^{+}$. We then deduce from (10) that $Q_{1}(t)$ explodes as $t \rightarrow T^{+}$. Thus

$$
t^{*}:=\sup \left\{t \in\left[0, T^{+}\right) ;\left\|Q_{1}(s)\right\| \leqslant 1, \forall s \in[0, t]\right\}
$$

belongs to $\left(0, T^{+}\right)$and $\left\|Q_{1}\left(t^{*}\right)\right\|=1$. Now we have

$$
\begin{aligned}
1 & =\left\|Q_{1}\left(t^{*}\right)\right\|=\left\|\int_{0}^{t^{*}}\left(-Q_{1}(s)^{2}+Q_{2}(s)^{2}+V^{\prime \prime}\left[x_{c}(s)\right]\right) d s\right\| \text { by (9) } \\
& \leqslant \int_{0}^{t^{*}}\left(\left\|Q_{1}(s)\right\|^{2}+b^{2} e^{4 \int_{0}^{t}\left\|Q_{1}(s)\right\| d s}+\left\|V^{\prime \prime}\left[x_{c}(s)\right]\right\|\right) d s \quad \text { by (10) } \\
& \leqslant t^{*}\left[1+b^{2} e^{4 t^{*}}+\left\|V^{\prime \prime}\right\|_{\infty}\right]<1 \quad \text { by (11) and (8) }
\end{aligned}
$$

which is a contradiction. Therefore $T^{+}>T^{*}$ for every $E \in C_{p w}^{0}\left(\mathbb{R}, \mathbb{R}^{N}\right)$.

Step 3: Conclusion. The same argument proves that $t^{*}>T^{*}$, i.e. $\left\|Q_{1}(t)\right\| \leqslant 1$ for every $t \in\left[0, T^{*}\right]$. Thus, by (91), (10) and (8) we have

$$
\left\|Q_{2}(t)-b I_{N}\right\|=\left\|\int_{0}^{t}\left[Q_{2} Q_{1}+Q_{1} Q_{2}\right](s) d s\right\| \leqslant 2 t b e^{2 t}<\frac{b}{2}, \forall t \in\left[0, T^{*}\right] .
$$




\subsection{Approximate solution}

We introduce the 'classical action' $S:(t, x) \in \mathbb{R} \times \mathbb{R}^{N} \rightarrow \mathbb{R}$

$$
S(t, x):=\int_{0}^{t}\left(\frac{1}{2}\left\|\dot{x}_{c}(s)\right\|^{2}-V\left[x_{c}(s)\right]\right) d s+\left\langle\dot{x}_{c}(t), x-x_{c}(t)\right\rangle
$$

and the approximate solution

$$
\begin{array}{cc}
\widetilde{\psi}(t, x):=\frac{b^{N / 4}}{C_{N}} \exp [\Phi(t, x)] \text { where } \\
\Phi(t, x):=\quad i\left(S(t, x)+\frac{1}{2}\left\langle Q(t)\left[x-x_{c}(t)\right], x-x_{c}(t)\right\rangle\right)+ \\
\left.\quad \int_{0}^{t}\left(i\left\langle x_{c}(s), E(s)\right\rangle-\frac{\operatorname{Tr}[Q(s)]}{2}\right) d s\right] .
\end{array}
$$

Note that equation (9) ensures that $\widetilde{\psi}(t) \in \mathcal{S}$. Indeed, for every $t \in\left(0, T^{*}\right)$,

$$
\begin{aligned}
\frac{d}{d t} \operatorname{det}\left[Q_{2}(t)\right] & =\operatorname{det}\left[Q_{2}(t)\right] \operatorname{Tr}\left[Q_{2}(t)^{-1} \dot{Q}_{2}(t)\right] \\
& =\operatorname{det}\left[Q_{2}(t)\right] \operatorname{Tr}\left[Q_{2}(t)^{-1}\left(-Q_{1}(t) Q_{2}(t)-Q_{2}(t) Q_{1}(t)\right)\right] \\
& =-2 \operatorname{Tr}\left[Q_{1}(t)\right] \operatorname{det}\left[Q_{2}(t)\right]
\end{aligned}
$$

which, together with (9), implies that

$$
\operatorname{det}\left[Q_{2}(t)\right]=b^{N} e^{-2 \int_{0}^{t} \operatorname{Tr}\left[Q_{1}(s)\right]} d s .
$$

Therefore,

$$
\begin{aligned}
\|\widetilde{\psi}(t)\|_{L^{2}\left(\mathbb{R}^{N}\right)} & =\frac{b^{N / 4}}{C_{N}} e^{-\frac{1}{2} \int_{0}^{t} \operatorname{Tr}\left[Q_{1}(s)\right] d s}\left(\int_{\mathbb{R}^{N}} e^{-\left\langle Q_{2}(t)\left[x-x_{c}(t)\right], x-x_{c}(t)\right\rangle} d x\right)^{1 / 2} \\
& =\frac{b^{N / 4}}{C_{N}} e^{-\frac{1}{2} \int_{0}^{t} \operatorname{Tr}\left[Q_{1}(s)\right] d s} \frac{C_{N}}{\operatorname{det}\left[Q_{2}(t)\right]^{1 / 4}}=1 .
\end{aligned}
$$

Proposition 3. There exists a constant $C_{*}>0$ such that, for every $V$ satisfying (2), $b>0, x_{0}, \dot{x}_{0} \in \mathbb{R}^{N}$ and $E \in C_{p w}^{0}\left(\mathbb{R}, \mathbb{R}^{N}\right)$, the solution $\psi$ of (1) with $\psi_{0}$ defined by (5) and the function $\widetilde{\psi}$ defined by (13) satisfy

$$
\|(\psi-\widetilde{\psi})(t)\|_{L^{2}\left(\mathbb{R}^{N}\right)} \leqslant C_{*}\left\|V^{(3)}\right\|_{\infty} \int_{0}^{t}\left\|Q_{2}(s)^{-1}\right\|^{3 / 2} d s, \quad \forall t \in\left[0, T^{*}\right],
$$

where $T^{*}$ is defined in Proposition [2.

\section{Proof of Proposition 3:}

Step 1: Equation satisfied by $\widetilde{\psi}$. On the one hand, we have (note that $S$ is linear in the variable $x$ )

$$
\begin{gathered}
\nabla \widetilde{\psi}=i\left(\nabla S+Q\left[x-x_{c}\right]\right) \widetilde{\psi}, \\
\Delta \widetilde{\psi}=\left[-\left\langle\nabla S+Q\left[x-x_{c}\right], \nabla S+Q\left[x-x_{c}\right]\right\rangle+i \operatorname{Tr}(Q)\right] \widetilde{\psi} \\
=\left[-\left\langle\dot{x}_{c}+Q\left[x-x_{c}\right], \dot{x}_{c}+Q\left[x-x_{c}\right]\right\rangle+i \operatorname{Tr}(Q)\right] \widetilde{\psi} .
\end{gathered}
$$

Thus

$$
\frac{1}{2} \Delta \widetilde{\psi}=\left[-\frac{1}{2}\left\|\dot{x}_{c}\right\|^{2}-\left\langle\dot{x}_{c}, Q\left[x-x_{c}\right]\right\rangle-\frac{1}{2}\left\langle Q^{2}\left[x-x_{c}\right],\left[x-x_{c}\right]\right\rangle+\frac{i}{2} \operatorname{Tr}(Q)\right] \widetilde{\psi} .
$$


On the other hand, we have

$$
\begin{aligned}
i \partial_{t} \widetilde{\psi}= & {\left[-\left(\partial_{t} S+\frac{1}{2}\left\langle\dot{Q}\left[x-x_{c}\right], x-x_{c}\right\rangle-\left\langle Q \dot{x}_{c}, x-x_{c}\right\rangle\right)-\frac{i}{2} \operatorname{Tr}(Q)-\left\langle x_{c}, E\right\rangle\right] \widetilde{\psi} } \\
= & {\left[-\frac{1}{2}\left\|\dot{x}_{c}\right\|^{2}+V\left[x_{c}\right]-\left\langle\ddot{x}_{c}, x-x_{c}\right\rangle+\left\|\dot{x}_{c}\right\|^{2}-\frac{1}{2}\left\langle\dot{Q}\left[x-x_{c}\right], x-x_{c}\right\rangle\right.} \\
& \left.+\left\langle Q \dot{x}_{c}, x-x_{c}\right\rangle-\frac{i}{2} \operatorname{Tr}(Q)-\left\langle x_{c}, E\right\rangle\right] \widetilde{\psi} \\
= & {\left[\frac{1}{2}\left\|\dot{x}_{c}\right\|^{2}+V\left[x_{c}\right]+\left\langle\nabla V\left(x_{c}\right)-E, x-x_{c}\right\rangle\right.} \\
& +\frac{1}{2}\left\langle\left[Q^{2}+V^{\prime \prime}\left(x_{c}\right)\right]\left[x-x_{c}\right], x-x_{c}\right\rangle+\left\langle Q \dot{x}_{c}, x-x_{c}\right\rangle \\
& \left.-\frac{i}{2} \operatorname{Tr}(Q)-\left\langle x_{c}, E\right\rangle\right] \widetilde{\psi}
\end{aligned}
$$

Combining (15) and (16) gives

$$
i \partial_{t} \widetilde{\psi}(t, x)+\frac{1}{2} \Delta \widetilde{\psi}(t, x)-V(x) \widetilde{\psi}(t, x)+\langle E(t), x\rangle \widetilde{\psi}(t, x)=r(t, x)
$$

where

$r(t, x):=-\left(V(x)-V\left(x_{c}\right)-\left\langle\nabla V\left(x_{c}\right), x-x_{c}\right\rangle-\frac{1}{2}\left\langle V^{\prime \prime}\left(x_{c}\right)\left[x-x_{c}\right], x-x_{c}\right\rangle\right) \widetilde{\psi}(t, x)$.

Step 2: Conclusion. Using (18), (13) and (14) and Taylor's formula, we get

$$
\begin{aligned}
\|r(t)\|_{L^{2}\left(\mathbb{R}^{N}\right)}^{2} & \leqslant \int_{\mathbb{R}^{N}}\left|\frac{\left\|V^{(3)}\right\|_{\infty}}{3 !}\left\|x-x_{c}\right\|^{3}\right|^{2} \frac{b^{N / 2}}{C_{N}^{2}} e^{-\left\langle Q_{2}(t)\left[x-x_{c}\right], x-x_{c}\right\rangle-\int_{0}^{t} \operatorname{Tr}\left(Q_{1}\right)} d x \\
& \leqslant \frac{\left\|V^{(3)}\right\|_{\infty}^{2}}{(3 !)^{2} C_{N}^{2}} \int_{\mathbb{R}^{N}}\left\|x-x_{c}\right\|^{6} e^{-\left\langle Q_{2}(t)\left[x-x_{c}\right], x-x_{c}\right\rangle} \sqrt{\operatorname{det}\left[Q_{2}(t)\right]} d x \\
& \leqslant C_{*}^{2}\left\|V^{(3)}\right\|_{\infty}^{2}\left\|Q_{2}(t)^{-1 / 2}\right\|^{6},
\end{aligned}
$$

where

$$
C_{*}:=\frac{1}{3 ! C_{N}}\left(\int_{\mathbb{R}^{N}}\|y\|^{6} e^{-\|y\|^{2}} d y\right)^{1 / 2} .
$$

Let $U(t, s)$ be the evolution operator for equation (11) (see Proposition 1). Then,

$$
(\psi-\widetilde{\psi})(t)=\int_{0}^{t} U(t, s) r(s) d s \text { in } L^{2}\left(\mathbb{R}^{N}\right), \forall t \in\left(0, T^{*}\right),
$$

and $U(t, s)$ is an isometry of $L^{2}\left(\mathbb{R}^{N}\right)$ for every $t \geqslant s \geqslant 0$, thus

$$
\|(\psi-\widetilde{\psi})(t)\|_{L^{2}\left(\mathbb{R}^{N}\right)} \leqslant \int_{0}^{t}\|r(s)\|_{L^{2}\left(\mathbb{R}^{N}\right)} d s \leqslant \int_{0}^{t} C_{*}\left\|V^{(3)}\right\|_{\infty}\left\|Q_{2}(s)^{-1}\right\|^{3 / 2} d s .
$$

Remark 1. The trajectory-coherent states $\widetilde{\psi}$ are only approximate solutions to the Schrödinger equation; however they are exact solutions for quadratic potentials $V$ (see (17) and (18)), which is the key point of reference [19]. 


\subsection{Proof of the main result}

Let $T^{*}=T^{*}\left(b,\left\|V^{\prime \prime}\right\|_{\infty}\right)>0$ be as in Proposition 2. The key point of the proof is the fact that, for every $E \in C_{p w}^{0}(\mathbb{R}, \mathbb{R})$, the approximate solution $\widetilde{\psi}$ has a Gaussian profile. Indeed, from (12), (13) and (14), one has

$$
|\widetilde{\psi}(t, x)|^{2}=\frac{\operatorname{det}\left[\sqrt{Q_{2}(t)}\right]}{C_{N}^{2}} e^{-\left\|\sqrt{Q_{2}(t)}\left[x-x_{c}(t)\right]\right\|^{2}},
$$

where $Q_{2}(t)$ is a real symmetric matrix satisfying (7).

Step 1: We prove that the set

$$
\begin{aligned}
& \mathcal{V}:=\{\phi \in \mathcal{S} ; \exists q \in \mathcal{S}_{N}^{+}(\mathbb{R}) \text { with } \sqrt{\frac{b}{2}} I_{N} \leqslant q \leqslant \sqrt{\frac{3 b}{2}} I_{N} \text { and } \\
&\left.\alpha \in \mathbb{R}^{N} \text { such that }|\phi(x)|^{2}=\frac{\operatorname{det}(q)}{C_{N}^{2}} e^{-\|q(x-\alpha)\|^{2}} \text { a.e. }\right\}
\end{aligned}
$$

is a strict closed subset of $\mathcal{S}$.

Clearly, $\mathcal{V}$ is a strict subset of $\mathcal{S}$. Let $\left(\phi_{n}\right)_{n \in \mathbb{N}}$ be a sequence of $\mathcal{V}$ that converges in $L^{2}\left(\mathbb{R}^{N}, \mathbb{C}\right)$ to $\phi_{\infty} \in \mathcal{S}$. For every $n \in \mathbb{N}$, we denote by $\alpha_{n} \in \mathbb{R}^{N}$ and $q_{n} \in \mathcal{S}_{N}^{+}(\mathbb{R})$ the corresponding parameters; $q_{n}$ satisfies

$$
\sqrt{\frac{b}{2}} I_{N} \leqslant q_{n} \leqslant \sqrt{\frac{3 b}{2}} I_{N}
$$

By extracting a subsequence if necessary, we may assume w.l.o.g. that $\phi_{n}(x) \rightarrow$ $\phi_{\infty}(x)$ for almost every $x \in \mathbb{R}^{N}$ (Lebesgue).

Step 1.1: Up to a possible extraction of a subsequence, we may assume that $q_{n} \rightarrow q_{\infty}$ where $q_{\infty} \in \mathcal{S}_{N}^{+}(\mathbb{R})$ and $\sqrt{\frac{b}{2}} I_{N} \leqslant q_{\infty} \leqslant \sqrt{\frac{3 b}{2}} I_{N}$. This may be seen by diagonalizing and appealing to the compactness of $O_{N}(\mathbb{R}) \times$ $[\sqrt{b / 2}, \sqrt{3 b / 2}]^{N}$.

Step 1.2: Up to a possible extraction of a subsequence, we may assume that $\alpha_{n} \rightarrow \alpha_{\infty} \in \mathbb{R}^{N}$. Working by contradiction, we assume that $\left(\alpha_{n}\right)_{n \in \mathbb{N}}$ is not bounded. We may assume w.l.o.g. that $\left\|\alpha_{n}\right\| \rightarrow+\infty$ when $n \rightarrow \infty$. Then, by (20)

$$
\left|\phi_{n}(x)\right|^{2}=\frac{\operatorname{det}\left(q_{n}\right)}{C_{N}^{2}} e^{-\left\|q_{n}\left(x-\alpha_{n}\right)\right\|^{2}} \leqslant \frac{(3 b / 2)^{N / 2}}{C_{N}^{2}} e^{-\frac{b}{2}\left\|x-\alpha_{n}\right\|^{2}} \rightarrow 0 \quad \text { a.e. } x \in \mathbb{R}^{N} .
$$

Thus $\phi_{\infty}=0$ (uniqueness of the a.e. limit), which is impossible because $\phi_{\infty} \in \mathcal{S}$.

Step 1.3: Conclusion. The uniqueness of the a.e. limit gives

$$
\left|\phi_{\infty}(x)\right|^{2}=\frac{\operatorname{det}\left(q_{\infty}\right)}{C_{N}^{2}} e^{-\left\|q_{\infty}\left(x-\alpha_{\infty}\right)\right\|^{2}} \quad \text { a.e. } x \in \mathbb{R}^{N}
$$

thus $\phi_{\infty} \in \mathcal{V}$. This concludes Step 1 .

Step 2: Let $\psi_{f} \in \mathcal{S} \backslash \mathcal{V}$ (which holds, in particular, when $\psi_{f}$ does not have a Gaussian profile). Then $\delta_{0}:=\operatorname{distance}_{L^{2}\left(\mathbb{R}^{N}, \mathbb{C}\right)}\left(\psi_{f} ; \mathcal{V}\right)>0$. Let

$$
T^{* *}=T^{* *}\left(\psi_{f}, b, V\right):=\min \left\{T^{*} ; \frac{\delta_{0}(b / 2)^{3 / 2}}{2 C_{*}\left\|V^{(3)}\right\|_{\infty}}\right\} .
$$


Then, using (17) and (19), we get that, for every $t \in\left[0, T^{* *}\right]$ and $E \in C_{p w}^{0}(\mathbb{R}, \mathbb{R})$, the solution $\psi$ of (1) satisfies

$$
\begin{aligned}
\left\|\psi_{f}-\psi(t)\right\|_{L^{2}(\mathbb{R})} & \geqslant\left|\left\|\psi_{f}-\widetilde{\psi}(t)\right\|_{L^{2}(\mathbb{R})}-\|(\widetilde{\psi}-\psi)(t)\|_{L^{2}(\mathbb{R})}\right| \\
& \geqslant \delta_{0}-C_{*}\left\|V^{(3)}\right\|_{\infty} \frac{t}{(b / 2)^{3 / 2}} \geqslant \frac{\delta_{0}}{2} .
\end{aligned}
$$

Acknowledgements: The authors were partially supported by the "Agence Nationale de la Recherche" (ANR) Projet Blanc EMAQS number ANR-2011BS01-017-01 and by the ERC advanced grant 266907 (CPDENL) of the 7th Research Framework Programme (FP7).

\section{References}

[1] Andrei Agrachev and Thomas Chambrion. An estimation of the controllability time for single-input systems on compact Lie groups. ESAIM Control Optim. Calc. Var., 12(3):409-441 (electronic), 2006.

[2] Vladislav G. Bagrov, Vladimir V. Belov, and Igor M. Ternov. Quasiclassical trajectory-coherent states of a particle in an arbitrary electromagnetic field. J. Math. Phys., 24(12):2855-2859, 1983.

[3] Vladislav G. Bagrov, Vladimir V. Belov, and Andrey Yu. Trifonov. Semiclassical trajectory-coherent approximation in quantum mechanics. I. High-order corrections to multidimensional time-dependent equations of Schrödinger type. Ann. Physics, 246(2):231-290, 1996.

[4] Karine Beauchard and Morgan Morancey. Local exact controllability of bilinear 1D Schrödinger equations and minimal time. Math. Control Relat. Fields, To appear, preprint, 2012, arXiv:1208.5393.

[5] Vladimir V. Belov and Margarita Kondratieva. Aharonov-bohm effect for nonstationary quasi-classical trajectory-coherent states in a homogeneous magnetic field. Russ. Phys. J., 10:83-90, 1992.

[6] Vladimir V. Belov and Margarita Kondratieva. Quasi-classical trajectorycoherent states of an anharmonic oscillator. Russ. Phys. J., 36:826-832, 1994.

[7] Ugo Boscain, Marco Caponigro, Thomas Chambrion, and Mario Sigalotti. A weak spectral condition for the controllability of the bilinear Schrödinger equation with application to the control of a rotating planar molecule. Comm. Math. Phys., 311(2):423-455, 2012.

[8] Ugo Boscain, Thomas Chambrion, and Mario Sigalotti. On some open questions in bilinear quantum control. arXiv:1304.7181, 2013.

[9] Nabile Boussaïd, Marco Caponigro, and Thomas Chambrion. Small time reachable set of bilinear quantum systems. 51st IEEE Conference on Decision and Control, pages 1083-1087, 2012.

[10] Rémi Carles. Nonlinear Schrödinger equation with time dependent potential. Commun. Math. Sci., 9(4):937-964, 2011. 
[11] Thomas Chambrion, Paolo Mason, Mario Sigalotti, and Ugo Boscain. Controllability of the discrete-spectrum Schrödinger equation driven by an external field. Ann. Inst. H. Poincaré Anal. Non Linéaire, 26(1):329-349, 2009.

[12] John W. Clark, Dennis G. Lucarelli, and Tzyh J. Tarn. Control of quantum systems. Int. J. Mod. Phys. B, 17:5397-5412, 2003.

[13] Monique Combescure and Didier Robert. Coherent states and applications in mathematical physics. Theoretical and Mathematical Physics. Springer, Dordrecht, 2012.

[14] Jean-Michel Coron. On the small-time local controllability of a quantum particle in a moving one-dimensional infinite square potential well. $C . R$. Math. Acad. Sci. Paris, 342(2):103-108, 2006.

[15] Domenico D'Alessandro. Small time controllability of systems on compact Lie groups and spin angular momentum. J. Math. Phys., 42(9):4488-4496, 2001.

[16] Daisuke Fujiwara. A construction of the fundamental solution for the Schrödinger equation. J. Analyse Math., 35:41-96, 1979.

[17] Garng M. Huang, Tzyh J. Tarn, and John W. Clark. On the controllability of quantum-mechanical systems. J. Math. Phys., 24(11):2608-2618, 1983.

[18] Paolo Mason and Mario Sigalotti. Generic controllability properties for the bilinear Schrödinger equation. Comm. Partial Differential Equations, 35(4):685-706, 2010.

[19] Mazyar Mirrahimi and Pierre Rouchon. Controllability of quantum harmonic oscillators. IEEE Trans. Automat. Control, 49(5):745-747, 2004.

[20] Didier Robert. Propagation of coherent states in quantum mechanics and applications. In Partial differential equations and applications, volume 15 of Sémin. Congr., pages 181-252, Paris, 2007. Soc. Math. France.

[21] Holger Teismann. Generalized coherent states and the control of quantum systems. J. Math. Phys., 46(12):122106, 13, 2005. 